\title{
A Survey of Stakeholder Perceptions towards Pre-Exposure Prophylaxes and Prospective HIV Microbicides and Vaccines in Kenya
}

Prince Bahati Ngongo $^{1 *}$, Judie Mbogua ${ }^{1}$, Jack Ndegwa ${ }^{2}$, George Githuka $^{3}$, Bonnie Bender ${ }^{1}$ and Florence Manguyu ${ }^{1}$

${ }^{1}$ International AIDS Vaccine Initiative, Kenya

${ }^{2}$ Kenya AIDS NGOs Consortium, Kenya

${ }^{3}$ National AIDS STI and Control Program, Kenya

\begin{abstract}
Understanding potential users' preferences for new HIV prevention options is an important component in designing effective roll-out and uptake plans. We surveyed opinions of 164 opinion leaders, health workers and key population representatives in five regions in Kenya to examine their preferences, concerns, perceived benefits and recommendations on Pre-exposure prophylaxes (PrEP) and potential HIV microbicides and vaccines. Data was captured via questionnaires and focus group discussions. We coded emerging themes in the data then extracted representative quotes. Cost, accessibility and efficacy were key determinants of acceptability and demand across prevention modalities. Myths, misconceptions, product associated stigma and anticipated societal resistance were identified as potential barriers to uptake, though the specifics of these varied from product to product. Vaccines elicited specific fears related to product-induced HIV infection and infertility. There was a strong preference for injectable over oral delivery of vaccines. There was also the perception that vaccines were more 'socially acceptable' and carried less stigma and concern around disinhibition than the other products. For microbicides, most groups favored the self-administered gel over ring or film because of individual's ability to control when to stop in case of side effect; however, health care providers showed a preference for the ring. Respondents expressed concern over potential sideeffects and the stigmatization of product users. With PrEP, there was a difference of opinion on the preferred mode of administration, with health care providers and MSM preferring oral PrEP and other respondents opting for longlasting injectable. Perceived benefit of PrEP was its demonstrated ability to protect against HIV. The primary concern associated with PrEP was safety and side effects. These included anxieties about the risk of taking drugs when one was not sick. Product design needs to proactively take into account community preferences and provide options that fit individual preferences as well as socio-cultural contexts.
\end{abstract}

Keywords: Community preferences on HIV prevention; New HIV preventions technologies; HIV target product profile

\section{Introduction}

Over the past 5 years, a significant amount of data has been released about new prevention technologies (NPT's) against HIV, specifically vaccines, microbicides and pre-exposure prophylaxes (PrEP). These trial findings, both positive and negative, are shaping community perceptions, expectations and preferences well in advance of proof of efficacy or product licensure. Interpretation of scientific results is complex and initial perceptions may have a long-lasting impact on uptake of eventual products. For instance, following the halting of the STEP vaccine trial, concerns were raised that the vaccine candidate increased susceptibility to HIV infection [1] these concerns may have created similar perceptions around non-STEP products. While the CAPRISA 004 microbicide trial demonstrated proof of concept that a vaginal microbicide gel can protect against HIV [2], the VOICE trial found no protection [3]. Similarly, the Fem-PrEP trial results found that oral ARV pre-exposure prophylaxis (PrEP) that worked in MSM appeared ineffective for heterosexual women [4]. While we now know that lack of efficacy in the Fem-PrEP study was largely due to lack of adherence [5], the initial conflicting data may have a long-lasting impact on the perception of product effectiveness at the community level.

As product and trial designs evolve, community perceptions, expectations and preferences for prospective microbicides, PrEP and HIV vaccines are also evolving. They are being shaped by community needs, as well as by trial results and the quality of communication around those results. Social marketing of family planning methods in Yemen demonstrated that successful introduction and use of contraceptive products is often affected by both individual and community perceptions, as well as preferences for modes of administration and service delivery [6]. Similarly, effective global family planning programs have shown that understanding community and individual product preferences is critical to acceptance and use of products [7].

A survey by Rudy et al. [8] found that potential barriers to uptake of a future HIV vaccine in Los Angeles included fear of vaccine-induced HIV infection, effects on reproduction, fear of injection, gender roles and power dynamics, HIV stigma, discrimination, affordability, and mistrust. In Uganda, Bishai et al. [9] found household wealth, price, and risk behavior were significant determinants of individual demand for a preventive HIV vaccine. They also found that demand was equally high for both low-efficacy and high-efficacy vaccines; this was largely due to the magnitude of the epidemic and personal experiences of respondents.

A survey by Kathleen [10] in the US found that a microbicide's product characteristics, HIV protective properties and contraceptive

*Corresponding author: Prince Bahati Ngongo, International AIDS Vaccine Initiative, Kenya, Tel: +254719043000; +254707367951; E-mail: PBahati@iavi.org

Received February 27, 2017; Accepted March 14, 2017; Published March 21 2017

Citation: Ngongo PB, Mbogua J, Ndegwa J, Githuka G, Bender B, et al. (2017) A Survey of Stakeholder Perceptions towards Pre-Exposure Prophylaxes and Prospective HIV Microbicides and Vaccines in Kenya. J AIDS Clin Res 8: 678, doi: 10.4172/2155-6113.1000678

Copyright: (c) 2017 Ngongo PB, et al. This is an open-access article distributed under the terms of the Creative Commons Attribution License, which permits unrestricted use, distribution, and reproduction in any medium, provided the original author and source are credited. 
properties were all significantly associated with willingness to use the product. They also found that women who ranked lower on socioeconomic indices (i.e., income, employment, education) appeared less concerned about product characteristics than those with higher socioeconomic ranking.

A survey by Galea et al. [11] in Peru followed by efficacy and lastly, potential side effects. Findings also revealed that potential sexual risk disinhibition, stigma and discrimination associated with PrEP use and mistrust of health care providers were also concerns found that PrEP acceptability ranged from 19.8 to $82.5 \%$, with out-of-pocket cost having the greatest impact on acceptability

The above studies did not evaluate PrEP, microbicides and vaccines as part of a combination package, or look at preferences for different regimens, delivery strategies among specific populations (e.g. sex workers, men who have sex with men, youth) and health care providers. The goal of this survey was to document individual product preferences as well as preferences among specific sub-groups. It was designed to identify factors that would facilitate or hinder acceptance and use. Specific questions that guided the survey included: what product traits (e.g. mode of delivery, frequency) do you consider preferable? What minimum level of efficacy level would be acceptable for introduction in your community? What price-point would be acceptable for introduction in your community? What do you perceive to be the barriers and facilitating factors for uptake in your community?

\section{Methods}

This study was conducted in Kenya between March and December 2012. Participants from NGOs were selected by the Kenya AIDS NGO Consortium (KANCO), a national network of more than 500 NGO's working on issues related to HIV/AIDS, based on consensus and availability. Health care providers were selected by the National AIDS and STIs Control Program, the government body responsible for technical coordination of HIV and AIDS services in Kenya, based on availability and regional representation. Representatives of key populations were selected by representative civil society organizations based on consensus, and availability. Key populations included female sex workers (FSW), men who have sex with men (MSM), and youth (14-24 years).

Participants were invited to attend standardized workshops on the status of research on PrEP, microbicides and HIV vaccines. A questionnaire (see Appendix) was administered to collect sociodemographic data and self-reported knowledge. Audio-taped and transcribed Focus Group Discussions (FGD) was held after the workshop to examine opinions, preferences and recommendations on PrEP, microbicides and vaccines. Two researchers independently listened to audiotaped FGD and validated the FGD notes. Results were shared and discrepancies resolved through discussion. Analysis continued through an iterative process of discussions to refine major themes. Data were grouped and frequencies used to determine common responses. Quantitative data collected on paper questionnaires was entered electronically using Microsoft Access 2007. Analysis was run using Stata version 11 (StataCorp LP, College Park, TX, USA). Frequencies and percentages are used to present categorical variables. Price data are reported using median and inter-quartile range (IQR).

\section{Results}

We conducted a total of 9 workshops for a total of 164 participants. Following the workshops, we held 8 FGDs. Participants were 53\% female and $47 \%$ male. $62 \%$ of the respondents primarily identified themselves as NGO representatives, $19 \%$ as Key Populations (MSM or Sex workers), $10 \%$ as health care providers and $9 \%$ as youth or adolescents. $27 \%$ also self-identified as HIV/AIDS or research advocates, $19 \%$ as community leaders or CAB members, $12 \%$ as research staff. The majority of respondents (58\%) indicated that they have been involved in HIV/AIDS advocacy for more than 5 years. There was less experience with research with $35 \%$ of respondents reporting involvement in HIV research for less than a year and just $20 \%$ for 5 years or more. Overall, self-reported knowledge on NPT research and results was high, approximately $70 \%$ of respondents reported having very or fairly good knowledge of research on PrEP, microbicides and HIV vaccines (Table 1 ).

We found that certain community preferences and expectations were shared across the NPTs evaluated. For instance, high efficacy, low cost and accessibility were identified as important determinants of acceptability and demand in focus group discussions. In addition, product associated stigma was negatively correlated with acceptability. Myths, misconceptions and anticipated societal resistance were identified as potential barriers to uptake, though the specifics of these varied from product to product.

Respondents were asked what would be the minimum level of efficacy necessary for them to advocate for introduction and use in their community. Across products, respondents indicated that approximately $75 \%$ efficacy would be required in order to recommend use.

Although not statistically significant, stratified data by groups of respondents show that both health care providers and sex workers were more willing to use or recommend products of lower efficacy, $57 \%$ and $69 \%$, respectively.

When asked about service delivery points, hospitals and health centers were considered the preferred facilities for accessing PrEP, microbicides and HIV vaccines; this was followed by family planning clinics and HIV counseling and testing facilities. 79\% of the respondents endorsed using the routine childhood immunization for HIV vaccine delivery. All MSM queried expressed interest in over the counter access for PrEP, but only $20 \%$ for microbicides. $67 \%$ of FSW supported OTC access to microbicides and $60 \%$ supported OTC for PreP. Among healthcare providers, support for OTC access was $60 \%$ and $50 \%$ for microbicides and PrEP, respectively (Table 2).

HIV vaccine specific feed-back included a strong preference for injectable over oral delivery. The preference for injectable was somewhat in contrast to respondents' feedback that 'fear of needles'

\begin{tabular}{|l|c|c|c|c|}
\hline Groups of respondents & Number & Female & Male & Missing Gender Data \\
\hline NGO representative & 102 & 52 & 41 & 9 \\
\hline Health Care Providers & 16 & 9 & 6 & 1 \\
\hline MSM & 16 & & 16 & \\
\hline Female Sex Workers & 16 & 16 & & \\
\hline Youth and adolescents & 14 & 5 & 9 & \\
\hline Total & 164 & 80 & 70 & 14 \\
\hline
\end{tabular}

Table 1: Distribution of respondents.

\begin{tabular}{|l|c|c|c|}
\hline & Mean & Median (IQR) & N \\
\hline HIV Vaccine & 74 & $80.0(60.0,90.0)$ & 118 \\
\hline Microbicides & 71 & $75.0(60.0,90.0)$ & 117 \\
\hline PrEP & 76 & $80.0(60.0,90.0)$ & 114 \\
\hline
\end{tabular}

Table 2: Recommended level of product efficacy by respondents. 
may be a barrier to uptake. Not surprisingly, respondents favored a product regimen that minimized the number of administrations over time.

Respondents identified a number of perceived benefits to HIV vaccines; these included the perception that vaccines are inherently effective and have the ability to confer life-long protection. Some commented that they are administered 'once' - pointing to experience based on some of the nationally administered vaccines through the Expanded Program of Immunizations (EPI) and the benefit of a product with a limited number of administrations. There was also the perception that vaccines had more 'social acceptability'. Vaccines did not seem to elicit the same sort of concerns regarding stigma and disinhibition as microbicides and PrEP. For some, vaccines were seen as the only acceptable intervention with children and young people.

"You don't have to take it daily"

"They offer lifelong prevention which is good"

"Should be introduced in the child vaccination schedule so that every newborn get the vaccine because it will provide lifelong protection for the whole generation"

Vaccines also invoked very specific fears, myths and misconceptions among respondents. Some of these were specific to HIV, but others related to broader and persistent concerns about vaccinations in general.

"Some people may think they will be infected because of the belief that the vaccine has HIV in it"

"Vaccines are meant to decrease the African population by making the male infertile...or reduce their brain activity"

Microbicide-specific feedback included an overall preference for self-administered gel over ring or film. Health care providers showed a preference for the ring, which may be due to the belief that this modality would improve adherence. Participants indicated that the timing of use associated with the gel, specifically its relation to sex, was a plus. This may speak to a preference to think about and utilize prevention tools in conjunction with risk behaviors.

Respondents articulated a number of perceived benefits associated with microbicides. This included the perception that it would be easy to use and have minimal side effects. The potential for a microbicide to double as a lubricant was seen as positive. Participants envisioned the licensed product to be something that could easily and discreetly be carried around. A clear theme that emerged was the potential benefit for woman - and the perceived benefit of a female-controlled technology.

"I do not need a lot of instructions to actually apply gel"

"It will act as a protection and at the same time as a lubricant; for safer sex and prevent friction at intercourse"

"The (female) sex worker will have the advantage of protecting themselves without having to negotiate or explain like condom"

Participants expressed concerns associated with cost and availability. Others expressed concern over potential safety issues and side-effects, such as itchiness, allergy and skin irritation. Skepticism over effectiveness of the gel was mentioned 24 times in questionnaires and frequently mentioned in focus groups. Some even reported that their skepticism is associated with mixed results and communications around past microbicides trials.
"Does it really protect? For how long will they be protected?"

Another major theme was the potential for stigma, particularly associated with perceptions of promiscuity that might be attached to use.

"The uptake of microbicides and PrEP will be hindered due to perception that those opting to use them are lax in morals"

"(Education and religious) Institutions may find it difficult to contain young people, with the safe preventions available including gels that can be carried around - you can no longer talk to the youth about consequences"

With PrEP, there was a difference of opinion on mode of administration, with health care providers and MSM preferring oral PrEP and other groups opting for injectable. For product regimen, the majority of respondents preferred a twice weekly regimen over daily or before/after sex. The disinclination for a before/after sex regimen is in contrast with preferences for microbicides. This may be associated with the belief that a more frequent or regular regimen is needed for efficacy, as that is the timing associated with trials that demonstrated protection.

Perceived benefits included 'proven' ability to protect against HIV. This contrasted against statements associated with vaccines and microbicides, where respondents expressed skepticism that they might 'really work.' Participants also viewed PrEP as 'easy to use' and a prevention modality that would be particularly relevant for key populations.

"It does not require skilled attendant"

"It has good efficacy rate"

The primary concern associated with PrEP was safety and side effects. These included anxieties about the risk of taking drugs when one was not sick. Myths and misconceptions, mainly associated with ARV's in general, were prominent.

"A HIV positive lady who was taking ARVs lost her sight."

"Some do not believe in taking drugs when they are not sick."

Stigma was seen as an important concern and potential barrier to introduction and uptake. Similarly, drug burden and adherence were also seen as obstacles.

"Stigma associated with ARV - people might think that one is HIV positive"

"If taken daily - taking medication daily is always a burden; what happens if you miss taking the drug on a particular day?"

More so than with vaccines and microbicides, the issues of cost and availability were factors that were raised.

"Once available and effective, the demand will be high and supply may be low". rich".

"It might be expensive at introduction and only accessible to the

Respondents suggested varied pricing and payment mechanisms based on regimen required for protection. The average acceptable price of NPTs was on average USD 3.95\$ for vaccines USD 0.43 for microbicides and USD 0.23 for PrEP (Table 3). Most respondents believed that the government and donors should provide vaccines free of charge while PrEP and Microbicides could be shared responsibilities between government and individuals. 
Page 4 of 5

\begin{tabular}{|c|c|c|c|c|c|c|c|c|c|c|}
\hline \multirow[b]{2}{*}{ If required: } & \multicolumn{4}{|c|}{ Preventive AIDS vaccine } & \multicolumn{3}{|c|}{ Microbicides } & \multicolumn{3}{|c|}{ Pre-exposure prophylaxis } \\
\hline & $\begin{array}{c}\text { Twice every } \\
\text { year }\end{array}$ & Once a year & $\begin{array}{l}\text { Once every } \\
\text { five years }\end{array}$ & $\begin{array}{l}\text { Once in a } \\
\text { lifetime }\end{array}$ & $\begin{array}{l}\text { Before each } \\
\text { sexual act }\end{array}$ & Once daily & Weekly & $\begin{array}{l}\text { Before or } \\
\text { after each } \\
\text { sexual act }\end{array}$ & Once daily & Twice a week \\
\hline & $\begin{array}{c}\text { Median } \\
(I Q R)^{N}\end{array}$ & $\begin{array}{c}\text { Median } \\
(I Q R)^{N}\end{array}$ & $\begin{array}{c}\text { Median } \\
(I Q R)^{N}\end{array}$ & $\begin{array}{c}\text { Median } \\
(I Q R)^{N}\end{array}$ & $\begin{array}{c}\text { Median } \\
(I Q R)^{N}\end{array}$ & $\begin{array}{c}\text { Median } \\
(I Q R)^{N}\end{array}$ & $\begin{array}{l}\text { Median } \\
(I Q R)^{N}\end{array}$ & $\begin{array}{c}\text { Median } \\
(\text { IQR) }\end{array}$ & $\begin{array}{l}\text { Median } \\
\text { (IQR)N }\end{array}$ & $\begin{array}{c}\text { Median } \\
(I Q R)^{N}\end{array}$ \\
\hline Total & $\begin{array}{c}100 \\
(20,500)^{125}\end{array}$ & $\begin{array}{c}150 \\
(50,500)^{119}\end{array}$ & $\begin{array}{c}500 \\
(100,1000)^{119}\end{array}$ & $\begin{array}{c}500 \\
(125,1250)^{116}\end{array}$ & $\begin{array}{c}20 \\
(10,50)^{127}\end{array}$ & $\begin{array}{c}30 \\
(10,50)^{117}\end{array}$ & $\begin{array}{c}50 \\
(20,100)^{118}\end{array}$ & $\begin{array}{c}20 \\
(10,100)^{122}\end{array}$ & $\begin{array}{c}20 \\
(10,50)^{115}\end{array}$ & $\begin{array}{c}50 \\
(12.5,100)^{116}\end{array}$ \\
\hline
\end{tabular}

Table 3: Recommended median pricing based on regimen required for protection (Ksh).

\section{Discussion and Conclusion}

Understanding potential users' and community preferences for new HIV prevention options is an important first step to designing roll-out plans that will ensure acceptability and eventual uptake. This study surveys preferences and perceptions around PrEP, microbicides and vaccines and explores differences of opinions by potential users and service providers. It provides specific insights to the Kenyan context, which is likely informed by participants' experiences with the national health system 's history of roll-out of prevention, treatment and care. This survey supports and adds new dimensions to studies that evaluated community opinions on HIV vaccines and microbicides in other contexts such as Uganda and in the US. For instance, high efficacy, low cost and accessibility were also identified as important determinants of acceptability and demand in the US [10] and Uganda [9].

We found that while certain concerns, preferences and expectations were shared across PrEP, microbicides and HIV vaccines, there was significant variation from product to product and from population to population. We found similar community concerns in Kenya as in the US [8] including fear of vaccine-induced HIV infection and potential effects on reproduction. However unlike in the US, HIV vaccines were seen as less stigmatizing, gender neutral and did not raise questions of affordability, and mistrust. We also saw similarities with a study conducted in Uganda [10] that found high demand for both low and high efficacy vaccines. While we did not measure correlations between household wealth, price and risk behavior with individual demand for a preventive HIV vaccine, we saw a trend towards higher demand and acceptance for low vaccine efficacy amongst sex workers and health care providers in Kenya.

This study concurs with findings in the US [9] that product characteristics and protective properties would be key in acceptability and use of microbicides. However, unlike in the US, contraceptive properties did not emerge as a determinant of acceptability. This study highlights Kenya specific issues that may affect acceptability including myths, misconceptions, HIV stigma, gender dynamics and religious and cultural issues. This culture specific context provides important information for future nationally-defined roll out plans. Furthermore, this study highlights that while new types of microbicides designs such as rings and films, may be preferred by health care providers, they may not necessarily be well understood or preferred by potential users and key populations, further emphasizing the need for multiple options. Participants also envisioned the licensed product to be something that could easily and discreetly be carried around, which provides an important clue for future marketing and packaging. The expressed concerns associated with cost and availability of microbicides may be driven by experience with the female condoms, where cost is seen to be prohibitive for some and there are frequent stock-outs [12].

While we found similar concerns between Kenya and Peru [11] regarding PrEP acceptability, such as sexual risk disinhibition, potential side effects, and stigma and discrimination associated with use of PrEP, this study reveals high acceptability for PrEP as an important additional tool for Key Populations. It also sheds lights on the high value accorded to PrEP by communities as a result of its proof of protection, this contrasts with microbicides, where mixed communications regarding efficacy resulted in doubt around its ability to protect. The high concern on cost and availability of PrEP may, in part, be due to the history associated with ARV access in Kenya.

While we found that among healthcare providers, support for OTC access was $60 \%$ and 50\% for microbicides and PrEP respectively, other surveys of health care providers' perceptions in South Africa [13], found that the majority of health care providers ( $80 \%$ of pharmacists and $75 \%$ of nurses) were supportive of microbicides and felt that the product should be accessed over-the-counter in pharmacies and in retail stores.

While respondents in Kenya represented a wide variety of stakeholders, including community leaders, health care providers and potential users, their views may not represent their greater community. Responses may have been influenced by the current status of the field (e.g. known efficacy and availability of PreP vs. unknown efficacy, administration methods and regimen for vaccine and microbicides) and would vary with time and new data. Despite earlier pilot testing of the questionnaire, we found that many respondents did not understand multiple-choice questions, resulting in missing or unreliable data on some questions. This could be related to the level of education of some respondents. Questions with unreliable data were discarded or analyzed based on relevant qualitative responses. Further studies with higher number of respondents would provide further insights on preferences by populations and geographies.

This study was conducted prior to the ground breaking results of on-Demand Preexposure Prophylaxis in Men at High Risk for HIV1 infection, also known as Ipergay [14] and the PROUD study (Preexposure Option for reducing HIV in the UK: immediate or Deferred [15] that found efficacy of PrEP to be over $86 \%$. These levels of confirmed efficacy in real settings (although not conducted in Kenya) may affect future perspectives on PrEP preferences by MSM. Further studies should explore the impact of PrEP demonstration projects on attitudes and perspectives of MSM and other key populations such as sex workers and adolescent and young women.

Work to understand evolving community preferences should be conducted to inform product design, clinical trial design and design of demonstration projects. Community engagement and advocacy programs need to proactively engage with researchers and communities to develop partnerships on target product profiles that provide options for individuals while maximizing the potential for public health impact for the communities. Longitudinal studies on factors that affect acceptability of new HIV prevention options at the individual and community levels are needed to inform design of better and scalable new HIV prevention products.

\section{Acknowledgement}

We would like to thank the team of KANCO trainers and facilitators, participants, 
Citation: Ngongo PB, Mbogua J, Ndegwa J, Githuka G, Bender B, et al. (2017) A Survey of Stakeholder Perceptions towards Pre-Exposure Prophylaxes and Prospective HIV Microbicides and Vaccines in Kenya. J AIDS Clin Res 8: 678. doi: 10.4172/2155-6113.1000678

NGOs and healthcare institutions that contributed to this survey. Special thanks go to our socio-behavioral expert, Sagri Singh, for her review and design of survey tools and FGDs, and our statistician Hellen Gathaka for conducting the data analysis.

IAVI's work is made possible by generous support from many donors including: the Bill and Melinda Gates Foundation; the Ministry of Foreign Affairs of Denmark Irish Aid; the Ministry of Finance of Japan; the Ministry of Foreign Affairs of the Netherlands; the Norwegian Agency for Development Cooperation (NORAD); the United Kingdom Department for International Development (DFID), and the United States Agency for International Development (USAID). The full list of IAVI donors is available at http://www.iavi.org. This study was made possible by the generous support of the American people through USAID. The contents are the responsibility of the International AIDS Vaccine Initiative and do not necessarily reflect the views of USAID or the United States Government.

\section{References}

1. Susan $P$ Buchbinder, Mehrotra DV, Duerr A, Fitzgerald DW, Mogg R, et al (2008) Efficacy assessment of a cell-mediated immunity HIV-1 vaccine (the Step Study): A double-blind, randomised, placebo-controlled, test-of-concept trial. Lancet 372: 1881-1893.

2. Karim QA, Karim SSA, Frohlich JA, Grobler AC, Baxter C, et.al (2010) Effectiveness and safety of tenofovir gel, an antiretroviral microbicide, for the prevention of HIV Infection in women. Science 329: 1168-1174.

3. Marrazzo JM, Ramjee G, Richardson BA, Gomez K, Mgodi N, et al. (2015) Tenofovir-based pre-exposure prophylaxis for HIV infection among African women. N Engl J Med 372: 512.

4. Vanpouille C, Arakelyan A, Margolis L (2012) Microbicides: Still a long road to success. Trends Microbiol 20: 369-375.

5. Corneli AL, Jennifer D, Wang M, Taylor D, Ahmed K, et al. (2014) FEM-PrEP: Adherence patterns and factors associated with adherence to a daily oral study product for pre-exposure prophylaxis. J Acquir Immune Defic Syndr 66: 324-331.
6. Maries Stopes International (2008) Perceptions and realities Yemeni men and women and contraception.

7. Jonhs Hopkins School of Public Health (2008) Populations Report.

8. Rudy ET, Newman PA, Duan N, Kelly EM, Roberts KJ, et al. (2005) HIV vaccine acceptability among women at risk: Perceived barriers and facilitators to future HIV vaccine uptake. AIDS Educ Prev 17: 253-267.

9. Bishai D, Pariyo B, Ainsworth G, Hill K (2004) Determinants of personal demand for an AIDS vaccine in Uganda: Contingent valuation survey. Bull World Health Organ 82: 652-660.

10. Kathleen MM (2007) Willingness to use microbicides is affected by the importance of product characteristics, use parameters, and protective properties. J Acquir Immune Defic Syndr 45: 93-101.

11. Galea JT, Kinsler JJ, Salazar X, Lee SJ, Giron M, et al. (2011) Acceptability of pre-exposure prophylaxis (PrEP) as an HIV prevention strategy: Barriers and facilitators to PrEP uptake among at-risk Peruvian populations. Int J STD AIDS 22: 256-262.

12. Mags EB, Jennifer AS, Joanne EM (2012) Progress and challenges to male and female condoms use in South Africa. Sex Health 9: 51-58.

13. Ramjee G, Morar NS, Mtimkulu J, Mantell JE, Gharbaharan V (2007) Perceptions of vaginal microbicides as an HIV prevention method among health care providers in KwaZulu-Natal, South Africa. AIDS Res Ther 4: 7.

14. Jean-Michel M, Capitant C, Spire B, Pialoux G, Cotte L, et al. (2015) Ondemand pre-exposure prophylaxis in men at high risk for HIV-1 infection. N Engl J Med 373: 2237-2246.

15. McCormack S, Dunn DT, Desai M, Dolling DI, Gafos M, et al. (2016) Preexposure prophylaxis to prevent the acquisition of HIV-1 infection (PROUD) Effectiveness results from the pilot phase of a pragmatic open-label randomised trial. Lancet 387: 53-60. 